\title{
Clinical phenotypes of Poppers maculopathy and their links to visual and anatomical recovery
}

Laure B. Van Bol11,2, MD, Rengin A. Kurt ${ }^{1}, \mathrm{MD}$, Pearse A. Keane ${ }^{1}, \mathrm{MD}$, FRCOphth, Bishwanath Pal ${ }^{1}, \mathrm{MD}$, Sobha Sivaprasad ${ }^{1}, \mathrm{MD}, \mathrm{FRCOphth}$

1. NIHR Moorfields Biomedical Research Centre, London, United Kingdom

2. Department of Ophthalmology, ULB (Université Libre de Bruxelles), Erasme Hospital, Brussels, Belgium.

Meeting presentation: Accepted for presentation during the 2017 ARVO Annual Meeting, Global connections in vision research, May 7-11 2017, Baltimore, Maryland

Financial Support: None

No conflicting relationship exists for any author

Disclosures:

The research was supported by the National Institute for Health Research (NIHR) Biomedical Research Centre based at Moorfields Eye Hospital NHS Foundation Trust and UCL Institute of Ophthalmology. The views expressed are those of the author(s) and not necessarily those of the NHS, the NIHR or the Department of Health. Dr Keane is supported by a Clinician Scientist award (CS-2014-14-023) from the National Institute for Health Research (NIHR).

Corresponding author and reprint requests: Laure Van Bol, Moorfields Eye Hospital, 162 City Rd, London EC1V 2PD, United Kingdom. Email: laure.vanbol@moorfields.nhs.uk 
The deleterious effect of alkyl nitrites inhalation (known as "Poppers") on photoreceptors is a well-known fact but the nature of foveal damage and its relation to visual prognosis remain unclear. We have therefore conducted a retrospective study on a series of patients diagnosed with poppers maculopathy at Moorfields Eye Hospital, London, United Kingdom, between August 2011 and August 2016. Approval for data collection and analysis was obtained from the Institutional Review Board at Moorfields and the study adhered to the tenets of the Helsinki Declaration. Electronic patient records and correspondence were searched for the term 'poppers' and 'maculopathy'. We then individually reviewed all cases and selected patients who presented with outer retinal changes confined to the fovea in the context of poppers use. Other causes of foveal yellow spot were ruled out by anamnesis (no history of sun gazing or laser exposure was reported) and patients with other retinal condition (e.g. previous episodes of CSR or family history of retinal dystrophy were excluded). Snellen visual acuity at the initial and last visit, colour photographs ( $n=27$ patients), optical coherence tomography (OCT) scans ( $n=39)$ and fundus autofluorescence (FAF) images ( $n=31)$ using Spectralis HRA-OCT (Heidelberg Engineering, Heidelberg, Germany) were evaluated and compared over time. We identified 39 patients who met our inclusion criteria. Amongst them, only 2 were women and the mean age was 39 years (ranging from 27 to 70). The mean follow-up time was 10 months (ranging from 1 to 84$)$.

From the OCT analysis, we observed 3 distinct phenotypes of maculopathy.

1. Subfoveal disturbance of the ellipsoid layer The most commonly encountered change was characterized by a "fuzzy" aspect of the ellipsoid layer and was observed in 22 patients (56\%). This OCT pattern was associated with poppers consumption varying between single to occasional use with duration of up to 2 years in documented cases. The initial Snellen visual acuity range was between 20/16 and 20/30. 
Fundus colour pictures revealed bilateral foveolar yellow lesions in 8 patients, whereas the remaining 4 cases showed normal fundus appearance. FAF at presentation in 17 patients showed no abnormality. After drug cessation, 8 of these patients (36\%) reported a complete resolution of their symptoms, and this was associated with the recovery of a normal foveal anatomy on OCT. 7 (32\%) reported an improvement of their symptoms but showed only a partial resolution of the macular changes on OCT, whereas the remaining 7 patients did not demonstrate any visual or anatomical improvement over time (range of follow-up: 2-24 months).

\section{Vitelliform-like lesion}

13 of our patients (33\%) presented with a more diffuse foveal involvement. The initial Snellen visual acuities ranged between $20 / 60$ to $20 / 30$, with 4 patients presenting with the lowest initial visual acuities observed in the cohort (3 patients presenting with 20/40 and one patient with 20/60). Chronic use of poppers (min. once a week) was reported in at least 9 patients, with periods of exposure ranging from 2 to 15 years. 11 of these patients presented with bilateral yellow foveal spot on fundus examination (fundus photographs were not available in 2 cases). OCT was undertaken in all cases and demonstrated a subfoveal detachment associated with a hyperreflective area of thickening extending from the ellipsoid layer to the ELM. Each patient (except one) benefited from autofluorescence imaging. 3 patients had normal fundus autofluorescence whilst 9 (69\%) showed a central area of mildly increased autofluorescence. Although complete cessation of poppers was advised, 3 patients did not comply. Interestingly, amongst the individuals who managed to stop their consumption, none of them demonstrated a full resolution of their symptoms concomitant with a regression of the OCT changes over time. In fact, 8 patients did not show any visual or anatomical improvement, 2 patients reported no change to their symptoms but showed a partial regression of the macular changes on OCT, whereas 3 patients experienced an improvement of their symptoms and visual acuities, which 
was not associated with structural recovery.

\section{Microhole}

At presentation, 4 patients were diagnosed with an asymmetric well-defined subfoveal defect of the ellipsoid layer. Interestingly, this type of lesion was found in the 2 female patients. Out of the 4 patients, 2 reported the occurrence of symptoms after a single trial of poppers, while the other 2 did not provide any details about their consumption. The initial Snellen visual acuities ranged between 20/17 and 20/30. Fundus examination showed a bilateral foveal yellow spot in one patient and was normal in the other 3 . Fundus autofluorescence was available for 2 patients and showed no abnormality. The follow-up duration was short, ranging from 2 to 3 months and only one individual showed a partial resolution of the photoreceptor disruption over time.

Since 2010, numerous authors have stated a clear association between poppers inhalation and foveal photoreceptor damage $[1,2,3,4,5]$ but, to our knowledge, our case series is the largest reported in literature. The clinical picture classically described is the occurrence of a bilateral yellow central spot corresponding to a subfoveal disturbance of the photoreceptor outer segments on OCT [1]. However, our study shows that vitelliform-like lesions and microholes are also part of the spectrum of poppers-related foveal toxicity. Moreover, our study shows that vitelliform-like lesions tend to occur amongst chronic users and that a significant proportion of them had not improved over time despite drug cessation. The effect of prolonged poppers consumption has already been highlighted in several studies but the conclusions on the visual prognosis are contradictory $[3,4,5]$. Our study brings further confirmation that chronic use may be associated with more severe and permanent photoreceptor damage. As any retrospective study, we are aware that our work has shortcomings, mainly the selection of our cases which was based on the anamnesis and the clinical appearance. However, we think that the 
occurrence of these peculiar foveal changes following poppers consumption makes other causes of maculopathy less likely. Other limitations include self-reported frequency of consumption and the potential bias induced by the use of final visual acuity reporting. 


\section{References}

1. Gruener AM, Jeffries MAR, El Housseini $Z$ et al. Poppers maculopathy. Lancet 2014;384:1606.

2. Vignal-Clermont C, Audo I, Sahel JA et al. Poppers-associated retinal toxicity. $n$ engl $j$ med 2010;363: 1583-1585.

3. Isabelle Audo I, El Sanharawi M, Vignal-Clermont C et al. Foveal damage in habitual poppers users. Arch Ophthalmol. 2011;129:703-708.

4. Pahlitzsch M, Mai C, Joussen AM et al. Poppers maculopathy: Complete restitution of macular changes in OCT after drug abstinence. Semin Ophthalmol. 2016;31:479-84.

5. Davies AJ, Kelly SP, Naylor SG, et al. Adverse ophthalmic reaction in poppers users: case series of "poppers maculopathy". Eye 2012;26:1479-1486. 


\section{Figure 1.}

A. A case of subfoveal disturbance of the ellipsoid layer in a 47-year-old male presenting with decreased central vision (20/30 in both eyes) 48 hours after a single trial of poppers. Fundus photographs(a) demonstrate a subtle yellowish lesion in both fovea corresponding to a normal autofluorescent signal on fundus autofluorescence(b). SDOCT(c) at baseline shows a subfoveal area of disturbance of the reflectivity of the ellipsoid layer. 5 months after exposure, his visual acuity recovered to 20/16 in both eyes and a complete resolution of the foveal changes is observed on SD OCT(d). B. A case of vitelliform-like lesion in a 39-year-old male presenting with bilateral central scotoma and a vision of 20/60 in both eyes in a context of heavy poppers use (2-3 times a week for 4 years). Fundus photographs(a) showing bilateral yellow spots with subtle pigmentary clumping, corresponding to an area of enhanced autofluorescent signal surrounded by a ring of reduced autofluorescence on fundus autofluorescence(b). SDOCT at presentation(c), showing bilateral subfoveal detachment with a hyper-reflective area of thickening extending from the ellipsoid layer to the ELM. 24 months later, his vision remains stable and only a partial regression of the foveal changes is noticed on SD-OCT despite drug cessation(d).

C. A case of microhole in a 28 -year-old patient, presenting with 20/20 vision and symptoms of metamorphopsia occurring after a single trial of poppers. Fundus photographs(a), autofluorescence(b) and SD-OCT(c) at presentation. A welldefined defect at the level of the ellipsoid layer is demonstrated on the right side. Five months later, a partial resolution of this defect is highlighted on SD-OCT(d). 